\title{
Probing inhomogeneities in type II superconductors by means of thermal fluctuations, magnetic fields and isotope effects
}

\author{
T. Schneider \\ Physik-Institut der Universität Zürich, Winterthurerstrasse 190, \\ CH-8057 Zürich, Switzerland
}

\begin{abstract}
Type II superconductors, consisting of superconducting domains embedded in a normal or insulating matrix, undergo a rounded phase transition. Indeed, the correlation length cannot grow beyond the spatial extent of the domains. Accordingly, the thermodynamic properties will exhibit a finite size effect. It is shown that the specific heat and penetration depth data of a variety of type II superconductors, including cuprates, exhibit the characteristic properties of a finite size effect, arising from domains with nanoscale extent. The finite size scaling analysis reveals essential features of the mechanism. Transition temperature and superfluidity increase with reduced domain size. The combined finite size and isotope effects uncover the relevance of local lattice distortions.
\end{abstract}

Key Words: Type II superconductors, granularity, finite size effects, thermal fluctuations

To appear in J. Superc., Proceedings Dynamic Inhomogeneities in Complex Systems, June 2003, Bled

\section{INTRODUCTION}

Since the discovery of superconductivity in cuprates by Bednorz and Müller [1] a tremendous amount of work has been devoted to their characterization. The issue of inhomogeneities and their characterization is essential for several reasons, including: First, if inhomogeneity is an intrinsic property, a re-interpretation of experiments, measuring an average of the electronic properties, is unavoidable. Second, inhomogeneity may point to a microscopic phase separation, i.e. superconducting grains, embedded in a non-superconducting matrix. Third, there is neutron spectroscopic evidence for nanoscale cluster formation and percolative superconductivity in various cuprates [2,3]. Fourth, nanoscale spatial variations in the electronic characteristics have been observed in underdoped $\mathrm{Bi}_{2} \mathrm{Sr}_{2} \mathrm{CaCu}_{2} \mathrm{O}_{8+\delta}$ with scanning tunnelling microscopy (STM) [4-7]. They reveal a spatial segregation of the electronic structure into 3nm diameter superconducting domains in an electronically distinct background. On the contrary, a large degree of homogeneity has been observed by Renner and Fischer [8]. As STM is a surface probe the relevance of these observations for bulk and thermodynamic properties remains to be clarified. Fifth, in $\mathrm{YBa}_{2} \mathrm{Cu}_{3} \mathrm{O}_{7-\delta}, \mathrm{MgB}_{2}, 2 \mathrm{H}-\mathrm{NbSe} 2$ and $\mathrm{Nb}_{77} \mathrm{Zr}_{23}$ considerably larger grains have been established. The magnetic field induced finite size effect revealed lower bounds ranging from $L=182$ to $814 \AA$ [9]. Sixth, since the change of the lattice parameters upon oxygen isotope exchange is negligibly small, the occurrence of a significant change in the inhomogeneities spatial extent, will provide clear evidence for the relevance of local lattice distortions in mediating superconductivity [10].

Here we review the attempts to probe the granularity of type II superconductors by means of thermal fluctuations and magnetic fields $[9,10]$. It is well-known that systems of finite extent, i.e. isolated superconducting grains, undergo a rounded and smooth phase transition [11]. As in an infinite and homogeneous system the transition temperature $T_{c}$ is approached the correlation length $\xi$ increases strongly and diverges at $T_{c}$. However, when superconductivity is restricted to grains with length scale $L_{i}$ in direction $i, \xi_{i}$ cannot grow beyond $L_{i}$. In type II superconductors, exposed to a magnetic field $H_{i}$, there is an additional limiting length scale $L_{H_{i}}=\sqrt{\Phi_{0} /\left(a H_{i}\right)}$ with $a \approx 3.12$, related to the average distance between vortex lines [9]. Indeed, as the magnetic field increases, the density of vortex lines becomes greater, but this cannot continue indefinitely, the limit is roughly set on the proximity of vortex lines by the overlapping of their cores. Due to these limiting length scales the phase transition is rounded and occurs smoothly. Indeed, approaching $T_{c}$ from below the transverse correlation length $\xi_{i}^{t}$ in direction $i$ and its real space counterpart $\xi_{i}^{-}=\sqrt{\xi_{j}^{t} \xi_{k}^{t}}$, where $i \neq j \neq k$, increase strongly. However, due to the limiting length scales $L_{i}$ and $L_{H_{i}}=\sqrt{\Phi_{0} /\left(a H_{i}\right)}$, it is bounded and cannot grow beyond

$$
\left.\begin{array}{c}
\xi_{i}^{-}\left(t_{p}\right)=\xi_{0 i}^{-}\left|t_{p}\right|^{-\nu}=L_{i}, \\
\sqrt{\xi_{i}^{-}\left(t_{p}\right) \xi_{j}^{-}\left(t_{p}\right)}=\sqrt{\xi_{0 i}^{-} \xi_{0 j}^{-}}\left|t_{p}\right|^{-\nu}=\sqrt{\Phi_{0} /\left(a H_{k}\right)}=L_{H_{k}}, i \neq j \neq k,
\end{array}\right\}
$$

where $t_{p}=1-T_{p} / T_{c}$ and $\nu$ denotes the critical exponent of the correlation lengths. Beyond the mean-field approximation it differs from $\nu=1 / 2$ and $a \approx 3.12$ is a universal constant [9]. In this context it is important to recognize 
that the confinement effect of the magnetic field in direction $i$ on fluctuations within a region $L_{H_{i}}$ acts only in the plane perpendicular to the field. As a remnant of the singularity at $T_{c}$ the thermodynamic quantities exhibit a so called finite size effect, i.e. a maximum or an inflection point at $T_{p}$. Two limiting regimes, characterized by

$$
L_{H_{i}}=\sqrt{\frac{\Phi_{0}}{a H_{i}}}=\left\{\begin{array}{l}
<\sqrt{L_{j} L_{k}} \\
>\sqrt{L_{j} L_{k}}
\end{array}, i \neq j \neq k\right\},
$$

can be distinguished. For $L_{H_{i}}<\sqrt{L_{j} L_{k}}$ the magnetic field induced finite size effect limits $\sqrt{\xi_{j}^{-} \xi_{k}^{-}}$to grow beyond $L_{H_{i}}$, while for $L_{H_{i}}>\sqrt{L_{j} L_{k}}$ the superconducting grains set the limiting length scales. Since $L_{H_{i}}$ can be tuned by the strength of the applied magnetic field, both limits are experimentally accessible. $L_{H_{i}}<\sqrt{L_{j} L_{k}}$ is satisfied for sufficiently high and $L_{H_{i}}>\sqrt{L_{j} L_{k}}$ for low magnetic fields. Thus, the occurrence of a magnetic field induced finite size effect requires that the magnetic field and the length scales of the superconducting grains satisfy the lower bound $H_{i}>\Phi_{0} /\left(a L_{j} L_{k}\right)$. Since superconductors fall in the experimentally accessible critical regime into the 3D-XY universality class [25], with known critical exponents and critical amplitude combinations, we take these properties for granted [12]. They include the exponents

$$
\alpha=2-3 \nu=-0.013, \nu=0.671
$$

and the critical amplitude combinations

$$
k_{B} T_{c}=\frac{\Phi_{0}^{2}}{16 \pi^{3}} \frac{\xi_{0 k}^{-}}{\lambda_{0 i} \lambda_{0 j}}, \quad i \neq j \neq k, \quad A^{ \pm} V_{c}^{-}=\left(R^{ \pm}\right)^{3}, V_{c}^{-}=\xi_{0 i} \xi_{0 j} \xi_{0 k}
$$

where

$$
\frac{A^{+}}{A^{-}}=1.07, R^{-}=0.815
$$

$\alpha$ and $A^{ \pm}$are the critical amplitude of the specific heat singularity, $c=\left(A^{ \pm} / \alpha\right)|t|^{-\alpha}+B^{ \pm}$, while $\xi_{0 i}^{-}$and $\lambda_{0 i}$ are the critical amplitude of the correlation length and penetration depth in direction $i$. In the homogeneous system these length scales diverge as $\xi_{i}^{-}=\xi_{0 i}^{-}|t|^{-\nu}, \lambda_{i}=\lambda_{0 i}|t|^{-\nu / 2}$ and $V_{c}^{-}$is the correlation volume.

\section{SPECIFIC HEAT}

In Fig.1a we displayed the data of Roulin et al. [13] for the temperature dependence of the heat coefficient of a $\mathrm{YBa}_{2} \mathrm{Cu}_{3} \mathrm{O}_{6.9}$ single crystal with $T_{c}=92.56 \mathrm{~K}$ at various magnetic fields applied parallel to the c-axis $\left(H_{c}\right)$. As a remnant of the zero field singularity, there is for fixed field strength a broad peak adopting its maximum at $T_{p}$ which is located below $T_{c}$. As $T_{p}$ approaches $T_{c}$, the peak becomes sharper with decreasing $H$ and evolves smoothly to the zero-field singularity, smeared by the inhomogeneity induced finite size effect. Since $T_{p}$ decreases systematically with increasing field, condition (2) applies in the form $L_{H_{c}}<\sqrt{L_{a} L_{b}}$ and there is a magnetic field induced finite size effect. As the data of Roulin et al. [13] are rather dense and extend close to zero field criticality, a detailed finite size scaling analysis appears to be feasible. In Fig.1b we plotted the data in terms of $\left(c\left(T, H_{c}\right) / T-B^{-}\right) /\left(A^{-}|t|^{-\alpha} / \alpha\right)$ versus $t / H_{c}^{1 / 2 \nu}$. Apparently the data falls on a single curve, which is the finite size scaling function $g(y)$ defined in terms of

$$
\left(\frac{c(T)}{T}-\widetilde{B}^{-}\right) /\left(\widetilde{A}^{-}|t|^{-\alpha}\right)=g(y), y=t\left(\Phi_{0} /\left(a H_{c}\left(\xi_{0 a b}^{-}\right)^{2}\right)\right)^{1 / 2 \nu}
$$

A finite size effect requires that $g(-1)=1$ at $T_{p}$, where $y=y_{p}=-1$, while $g( \pm y \rightarrow 0) \propto|y|^{-|\alpha|}$ holds for $\alpha<0$ and $g( \pm y \rightarrow \infty)=1$. To qualify the remarkable collapse of the data we note that the estimates for $\widetilde{A}^{-}=A^{-} / \alpha$ and $B^{-}$ have been derived from the zero field data displayed in Fig.1a. The solid line for $t<0$ is $c / T=\widetilde{A}^{-}|t|^{-\alpha}+\widetilde{B}^{-}$with $\widetilde{A}^{-}=A^{-} / T_{c} \alpha=-0.073\left(\mathrm{~J} / \mathrm{K}^{2}\right.$ gat $), \quad \widetilde{B}^{-}=0.179\left(\mathrm{~J} / \mathrm{K}^{2}\right.$ gat $), \widetilde{B}^{+}=0.181\left(\mathrm{~J} / \mathrm{K}^{2}\right.$ gat $), T_{c}=92.56 \mathrm{~K}$ and the critical exponent $\alpha=-0.013$, to indicate the inhomogeneity induced deviations from the leading zero field critical behavior of perfect $\mathrm{YBa}_{2} \mathrm{Cu}_{3} \mathrm{O}_{6.9}$. Since the data collapse onto the finite scaling function has been achieved without any arbitrary fitting parameter, the existence of the magnetic field induced finite size effect is well confirmed. Furthermore, Fig.1a shows how the broad anomaly in the specific heat coefficient sharpens, while the maximum height at $T_{p}\left(H_{c}\right)$ increases 
with reduced field strength, evolving smoothly to the zero field peak, rounded by inhomogeneities. From the finite size scaling plot displayed in Fig.1b we also deduce that with $\xi_{0 a b}^{-}=14.4 \mathrm{~A}$, that at $y_{p}$, where $|t| / H_{c}^{1 / 2}=-0.013$, the scaling function is close to 1 , as required. Furthermore, the solid line which is $\left(c\left(T, H_{c}\right) / T-\widetilde{B}^{-}\right) /\left(\widetilde{A}^{-}|t|^{-\alpha}\right)=$ $0.957\left(-t / H_{c}^{1 / 2}\right)^{\alpha}$, confirms the divergence of the scaling function in the limit $\pm y \rightarrow 0$.
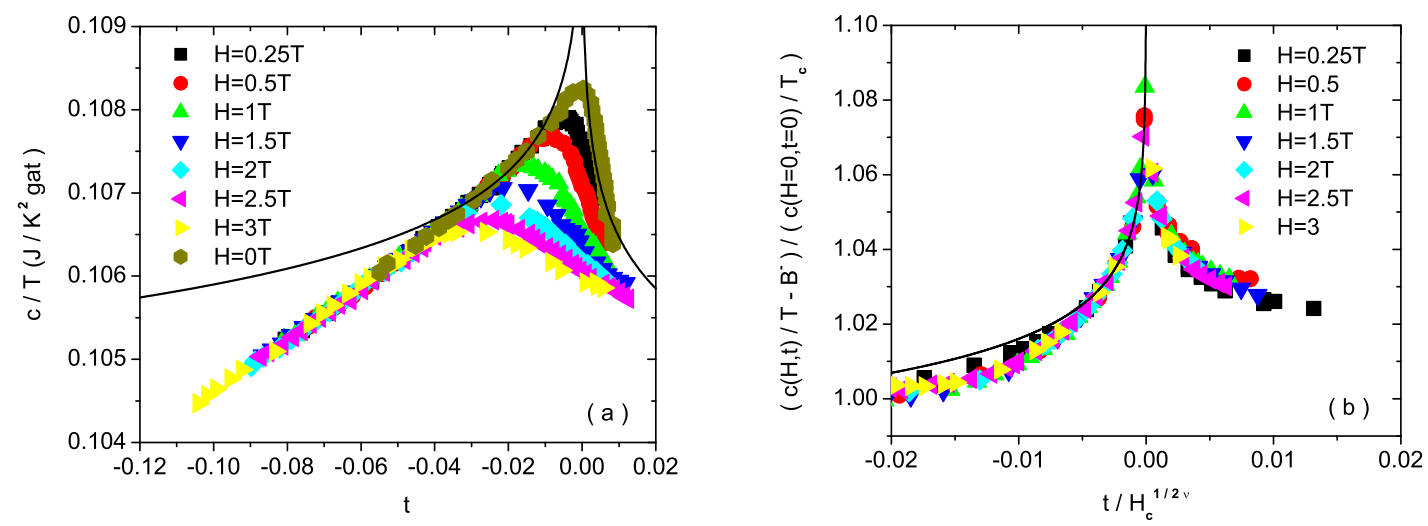

FIG. 1. (a): Specific heat coefficient $c / T$ versus $t$ of $\mathrm{YBa}_{2} \mathrm{Cu}_{3} \mathrm{O}_{6.9}$ with $T_{c}=92.56 \mathrm{~K}$ at various magnetic fields derived from the data of Roulin et al. [13]. The solid lines are $c / T=\widetilde{A}^{ \pm}|t|^{-\alpha}+\widetilde{B}^{ \pm}$with $\alpha$ and $A^{+} / A^{-}$given by Eqs.(3) and the parameters listed in the text. The deviations of the zero field data from these lines reveal around $t=0$ the finite size effect due to inhomogeneities; (b): $\left(c\left(T, H_{c}\right) / T-\widetilde{B}^{-}\right) /\left(\widetilde{A}^{-}|t|^{\alpha}\right)$ versus $t / H_{c}^{1 / 2 \nu}$ for $\mathrm{YBa}_{2} \mathrm{Cu}_{3} \mathrm{O}_{6.9}$ with $T_{c}=92.56 \mathrm{~K}$ derived from the data of Roulin et al. [13] with $H$ in $\mathrm{T}$. The solid line is $\left(c\left(T, H_{c}\right) / T-\widetilde{B}^{-}\right) /\left(\widetilde{A}^{-}|t|^{-\alpha}\right)=0.957\left(-t / H_{c}^{1 / 2}\right)^{\alpha}$ with $\alpha=-0.013$, confirming the divergence of the scaling function in the limit $\pm y \rightarrow 0$.

The shift of $T_{p}$ with increasing $H_{c}$ is according to Eq.(1) given by

$$
\left|t_{p_{i}}\right|=\left(\frac{a \xi_{0 i}^{-} \xi_{0 j}^{-} H_{k}}{\Phi_{0}}\right)^{1 / 2 \nu} .
$$

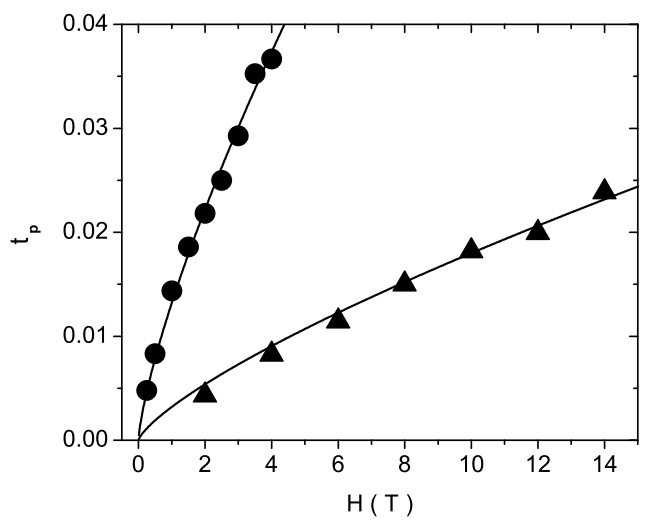

FIG. 2. $\left|t_{p}\right|$ versus $H_{c}(\bullet)$ and $H_{a b}(\boldsymbol{\Delta})$ for $\mathrm{YBa}_{2} \mathrm{Cu}_{3} \mathrm{O}_{6.9}$ with $T_{c}=92.56 \mathrm{~K}$ derived from the data of Roulin et al. [13]. The solid lines are $\left|t_{p}\left(H_{a b}\right)\right|=0.0033 H_{a b}^{1 / 2 \nu}$ and $\left|t_{p}\left(H_{c}\right)\right|=0.0132 H_{c}^{1 / 2 \nu}$ with $\nu$ listed in Eq.(3).

In Fig. 2 we show $\mathrm{t}_{p}$ versus $H_{a b}$ and $H_{c}$ for $\mathrm{YBa}_{2} \mathrm{Cu}_{3} \mathrm{O}_{6.9}$ single crystal with $T_{c}=92.56 \mathrm{~K}$ derived from the data of Roulin et al. [13]. The solid lines are $\left|t_{p}\left(H_{a b}\right)\right|=0.0033 H_{a b}^{1 / 2 \nu}$ and $\left|t_{p}\left(H_{c}\right)\right|=0.0132 H_{c}^{1 / 2 \nu}$ with $H$, revealing that the critical regime is attained. With $\nu$ given by Eq.(3) and $a=3.12$ these fits yield for the critical amplitudes the estimates 


$$
\sqrt{\xi_{0 a b}^{-} \xi_{0 c}^{-}}=5.7 \AA, \xi_{0 a b}^{-}=14.4 \AA, V_{c}^{-}=\left(\xi_{0 a b}^{-}\right)^{2} \xi_{0 c}^{-}=469 \AA^{3} .
$$

To estimate the correlation volume from the specific heat coefficient in terms of the universal relation, $A^{ \pm} V_{c}^{-}=$ $\left(R^{ \pm}\right)^{3}$ (Eq.(4) we note that $A^{-}=T_{c} \alpha \widetilde{A}^{-}, A^{-}\left(\mathrm{cm}^{-3}\right)=\left(10^{7} / k_{B} / V_{\text {gat }}\right) A^{-}\left(\mathrm{mJ} / \mathrm{K} / \mathrm{cm}^{3}\right), V_{\text {gat }}=8 \mathrm{~cm}^{3}$ and $\widetilde{A}^{-}=$ $-0.073\left(\mathrm{~J} / \mathrm{K}^{2}\right.$ gat $)$, corresponding to the solid line in Fig.1a, give $A^{-}=7.9610^{-4} \AA^{-3}$, yielding with $R^{-}=0.815$ (Eq.(5)) the correlation volume $V_{c}^{-} \approx 680 \AA^{3}$, which is reasonably close to the value derived from the magnetic field induced finite size effect (Eq.(8)). Since at the lowest attained fields $H_{c}=0.25 \mathrm{~T}$ and $H_{a b}=2 \mathrm{~T}$ a shift from $T_{c}$ to $T_{p}$ is still present (see Fig.1a), we derive from Eq.(2) for the length scales of the sample inhomogeneities the lower bounds

$$
L_{a b}>515 \AA, \quad \sqrt{L_{a b} L_{c}}>182 \AA .
$$

As in zero field the correlation volume $V_{c}^{-}\left|t_{p}\right|^{-3 \nu}$ cannot grow beyond the volume $V_{i}$ of the inhomogeneities, this limiting volume scale is obtained from $V_{i}=V_{c}^{-}\left|t_{p}\right|^{-3 \nu}$ and $t_{p}$ evaluated in zero field. With $V_{c}^{-}=469 \AA^{3}$ and $\left|t_{p}\right|=0.0025$ taken from Fig.1a, we obtain the estimates $V_{i}=7.510^{7} \AA^{3}$ and $V_{i}^{1 / 3}=422 \AA$ which is consistent with the aforementioned lower bounds.

Next we turn to $\mathrm{Nb}_{77} \mathrm{Zr}_{23}$ [14], $2 \mathrm{H}-\mathrm{NbSe}_{2}$ [15] and $\mathrm{MgB}_{2}$ [16], type II superconductors supposed to have comparative large correlation volumes. In Fig.3 we displayed $\left|t_{p}\right|$ versus $H$ derived from the respective experimental data. In contrast to the corresponding plot for nearly optimally $\mathrm{YBa}_{2} \mathrm{Cu}_{3} \mathrm{O}_{7-\delta}$ (Fig.2), the data points to a linear relationship. Consequently, the critical regime, where $\left|t_{p}\right| \propto H^{1 / 2 \nu}$ with $\nu \approx 2 / 3$ holds (Eq.(7)), is not attained. Nevertheless, there is clear evidence for a magnetic field induced finite size effect, because $T_{p}$ shifts monotonically to a lower value with increasing magnetic field. Since the data points to an effective critical exponent $\nu \approx 1 / 2$ which applies over an unexpectedly extended range, we use Eq.(7) with $\nu=1 / 2$ to derive estimates for the amplitude of the respective transverse correlation lengths and the correlation volume in terms of

$$
\left|t_{p}\right|=b_{i} H_{i}=\frac{\xi_{0 j}^{-} \xi_{0 k}^{-}}{L_{H_{i}}^{2}}=\frac{a H_{i} \xi_{0 j}^{-} \xi_{0 k}^{-}}{\Phi_{0}}
$$

with $a=3.12$.

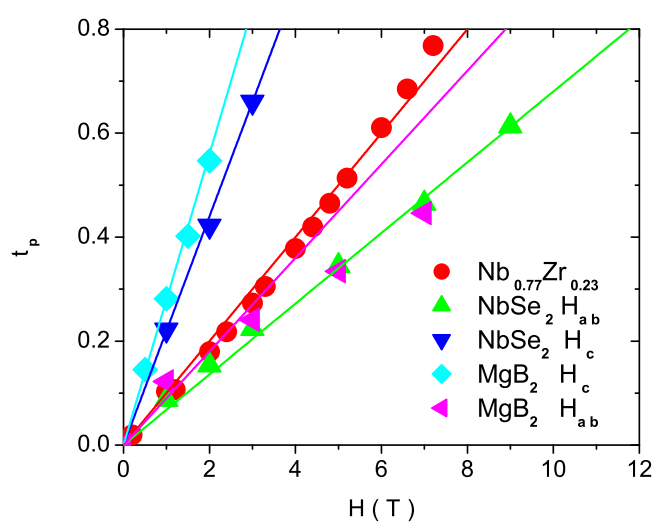

FIG. 3. $\left|t_{p}\right|$ versus $H$ for $\mathrm{Nb}_{77} \mathrm{Zr}_{23}$ [14], 2H-NbSe 2 [15] and $\mathrm{MgB}_{2}$ [16] derived from the respective references. The straight lines are Eq.(10) with the parameters listed in Table I.

The respective straight lines in Fig.3 are this relation with the parameters listed in Table I. For comparison we included the corresponding parameters for $\mathrm{YBa}_{2} \mathrm{Cu}_{3} \mathrm{O}_{6.9}$ [13], $\mathrm{YBa}_{2} \mathrm{Cu}_{3} \mathrm{O}_{6.6},(\mathrm{~K}, \mathrm{Ba}) \mathrm{BiO}_{3}[17]$ and $\mathrm{HgBa}_{2} \mathrm{Ca}_{2} \mathrm{Cu}_{3} \mathrm{O}_{7-\delta}$ [19] where the critical regime is attained. It is evident that $\mathrm{Nb}_{77} \mathrm{Zr}_{23}, 2 \mathrm{H}-\mathrm{NbSe}_{2}$ and $\mathrm{MgB}_{2}$ are type II superconductors with comparatively large correlation lengths. Compared to $\mathrm{YBa}_{2} \mathrm{Cu}_{3} \mathrm{O}_{6.9}$ and $\mathrm{YBa}_{2} \mathrm{Cu}_{3} \mathrm{O}_{6.6}$ the correlation volume is 3 orders of magnitude larger, which renders the amplitude of the specific heat singularity very weak (see Eq.(4). Nevertheless, the unambiguous evidence for the magnetic field induced finite size effect reveals that fluctuations, even though not critical, are at work. For this reason there is no critical line $T_{c 2}(H)$ of continuous phase transitions, but a line $T_{p}(H)$ where the specific heat peak, broadening and decreasing with increasing field, adopts its maximum value and the correlation length attains the limiting magnetic length scale $L_{H_{i}}$. Because the fluctuations are also 
subject to the finite size effect arising from inhomogeneities with length scale $L_{j}$, the magnetic finite size effect is observable as long as $\sqrt{L_{j} L_{k}}>L_{H_{i}}$. The resulting lower bounds for the length scale of inhomogeneities affecting the thermodynamic properties are also included in Table I. Noting that these bounds stem from studies where no attempt was made to explore the low field behavior, it is conceivable that the actual length scale of the inhomogeneities is much larger. To our best knowledge, the only absolute reference stems from the finite size scaling analysis of the zero field specific heat data of nearly optimally doped $\mathrm{YBa}_{2} \mathrm{Cu}_{3} \mathrm{O}_{7-\delta}$, where $L$ was found to range from 290 to $419 \AA$ $[25,21]$. Interestingly enough, the largest bound found here applies to the cubic superconducting alloy $\mathrm{Nb}_{77} \mathrm{Zr}_{23}$.

\begin{tabular}{|c|c|c|c|c|c|c|c|}
\hline & $\mathrm{T}_{c}(\mathrm{~K})$ & $\xi_{0}^{-}, \sqrt{\xi_{0 a b}^{-} \xi_{0 c}^{-}(\AA)}$ & $\xi_{0 a b}^{-}(\AA)$ & $\gamma$ & $\mathrm{V}_{c}^{-}\left(\mathrm{A}^{3}\right)$ & $\mathrm{L}, \mathrm{L}_{a b}(\AA)$ & $\sqrt{L_{a b} L_{c}}(\AA)$ \\
\hline $\mathrm{Nb}_{77} Z r_{23}$ & 10.8 & 55 & - & 1 & $1.710^{5}$ & $>814$ & - \\
\hline $2 \mathrm{H}-\mathrm{NbSe}_{2}$ & 7.1 & 43 & 94 & 4.8 & $1.710^{5}$ & $>258$ & $>258$ \\
\hline $\mathrm{MgB}_{2}$ & 35 & 52 & 110 & 4.5 & $2.910^{5}$ & $>364$ & $>258$ \\
\hline$(\mathrm{K}, \mathrm{Ba}) \mathrm{BiO}_{3}$ & 31.6 & 50 & - & 1 & $1.2510^{5}$ & $>258$ & - \\
\hline $\mathrm{YBa}_{2} \mathrm{Cu}_{3} \mathrm{O}_{6.95}$ & $\overline{92.6}$ & 5.7 & 14.4 & 6.4 & $4.710^{2}$ & $>515$ & $>182$ \\
\hline $\mathrm{YBa}_{2} \mathrm{Cu}_{3} \mathrm{O}_{6.6}$ & 64.2 & $(7.3)$ & 32.6 & $\overline{(20)}$ & $1.710^{3}$ & $>576$ & - \\
\hline $\mathrm{HgBa}_{2} \mathrm{Ca}_{2} \mathrm{Cu}_{3} \mathrm{O}_{7-\delta}$ & 111.1 & - & 12 & $(52)$ & - & $>346$ & - \\
\hline $\mathrm{Bi}_{2.12} \mathrm{Sr}_{1.71} \mathrm{Ca}_{1.22} \mathrm{Cu}_{1.95} \mathrm{O}_{8+\delta}$ & 85.7 & - & - & - & - & $<69$ & $<69$ \\
\hline
\end{tabular}

Table I:Summary of the estimates for the critical amplitudes of the correlation lengths, correlation volume $V_{c}^{-}=$ $\left(\xi_{0 a b}^{-}\right)^{2} \xi_{0 c}^{-}$, anisotropy $\gamma=\xi_{0 a b}^{-} / \xi_{0 c}^{-}$, lower bounds for the length scales $L_{a b}$ and $\sqrt{L_{a b} L_{c}}$ of inhomogeneities, derived from experimental data by means of the magnetic field induced finite size effect. For $\mathrm{YBa}_{2} \mathrm{Cu}_{3} \mathrm{O}_{6.6}$ we used $\gamma=20$, taken from Janossy et al. [20] to estimate $\xi_{0 a b}^{-}$.

On the contrary, the specific heat measurements reveal that in the Bismuth- and Thallium based cuprates $T_{p}$ appears not to shift up to $14 \mathrm{~T}$, for fields applied parallel or perpendicular to the c-axis [18,22,23]. To derive detailed estimates we consider the single crystal data for the specific heat coefficient of Junod et al. [23] for $\mathrm{Bi}_{2.12} \mathrm{Sr}_{1.71} \mathrm{Ca}_{1.22} \mathrm{Cu}_{1.95} \mathrm{O}_{8+\delta}$. To reduce artifacts of the huge background we displayed in Fig.4 the data in terms of $(c(H, T)-c(H=0, t)) / T$ versus $T$ for fields applied parallel to the c-axis. Apparently, there is no shift of the peak around $T_{p}=85.1 \mathrm{~K}$ with increasing magnetic field up to $H=14 \mathrm{~T}$. Noting that the same behavior was found for fields perpendicular to the c-axis [23], these data lead with inequality (2) to the upper bounds

$$
L_{a b}<69 \AA, \sqrt{L_{a b} L_{c}}<69 \AA,
$$

uncovering nanoscale superconducting grains, consistent with the length scale of the inhomogeneities observed with STM spectroscopy [4-7]. As STM is a surface probe, our analysis establishes that these grains are not merely an artefact of the surface, but a bulk property with spatial extent, giving rise to a rounded thermodynamic phase transition which occurs smoothly.

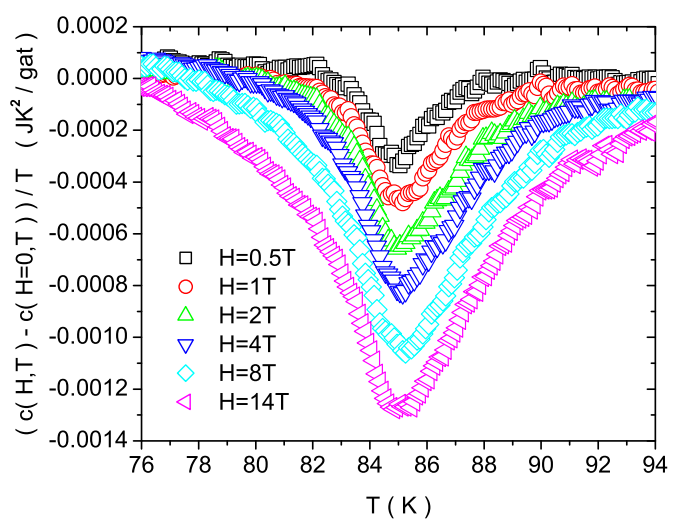

FIG. 4. $\left(c\left(H_{c}, T\right)-c\left(H_{c}=0, T\right)\right) / T$ versus $T$ of a $\mathrm{Bi}_{2.12} \mathrm{Sr}_{1.71} \mathrm{Ca}_{1.22} \mathrm{Cu}_{1.95} \mathrm{O}_{8+\delta}$ single crystal taken from Junod et al. [23]. The magnetic field is parallel to the c-axis 


\section{PENETRATION DEPTH}

Considering again the 3D-XY critical point, extended to the anisotropic case, the penetration depths and transverse correlation lengths in directions $i$ and $j$ are universally related by $[25,26]$

$$
\frac{1}{\lambda_{i}(T) \lambda_{j}(T)}=\frac{16 \pi^{3} k_{B} T}{\Phi_{0}^{2} \sqrt{\xi_{i}^{t}(T) \xi_{j}^{t}(T)}} .
$$

When the superconductor is inhomogeneous, consisting of superconducting grains with length scales $L_{i}$, embedded in a non-superconducting medium, $\xi_{i}^{t}$ does not diverge but is bounded by $\xi_{i}^{t} \xi_{j}^{t}=\left(\xi_{k}^{-}\right)^{2} \leq L_{k}^{2}$, where $i \neq j \neq k$. The resulting finite size effect is clearly seen in the microwave surface impedance data for $\lambda_{a b}^{2}(T=0) / \lambda_{a b}^{2}(T)$ versus $T$ of Jacobs et al. [24], displayed in Fig.5a. The solid curve indicates the leading critical behavior of the homogeneous system. A characteristic feature of this finite size effect is the occurrence of an inflection point at $T_{p} \approx 87 \mathrm{~K}$, giving rise to an extremum in $d\left(\lambda_{a b}^{2}(T=0) / \lambda_{a b}^{2}(T)\right) / d T$. Here Eq.(12) reduces to

$$
\frac{1}{\lambda_{a b}^{2}\left(T_{p}\right)} \approx \frac{1}{\lambda_{a}\left(T_{p}\right) \lambda_{b}\left(T_{p}\right)}=\frac{16 \pi^{3} k_{B} T_{p}}{\Phi_{0}^{2} L_{c}}
$$

With $\lambda_{a b}(T=0)=1800 \AA$ as obtained from $\mu \mathrm{SR}$ measurements [28], $T_{p} \approx 87 \mathrm{~K}$ and $\lambda_{a b}^{2}(T=0) / \lambda_{a b}^{2}\left(T_{P}\right)=0.066$ we find $L_{c} \approx 68 \AA$, consistent with lower bounds (11) derived from the specific heat data. Thus, although the superconducting grains are of nanoscale only, a very small correlation volume makes the critical regime attainable. Clear evidence for 3D-XY critical behavior was also observed in epitaxially grown Bi2212 films [27].
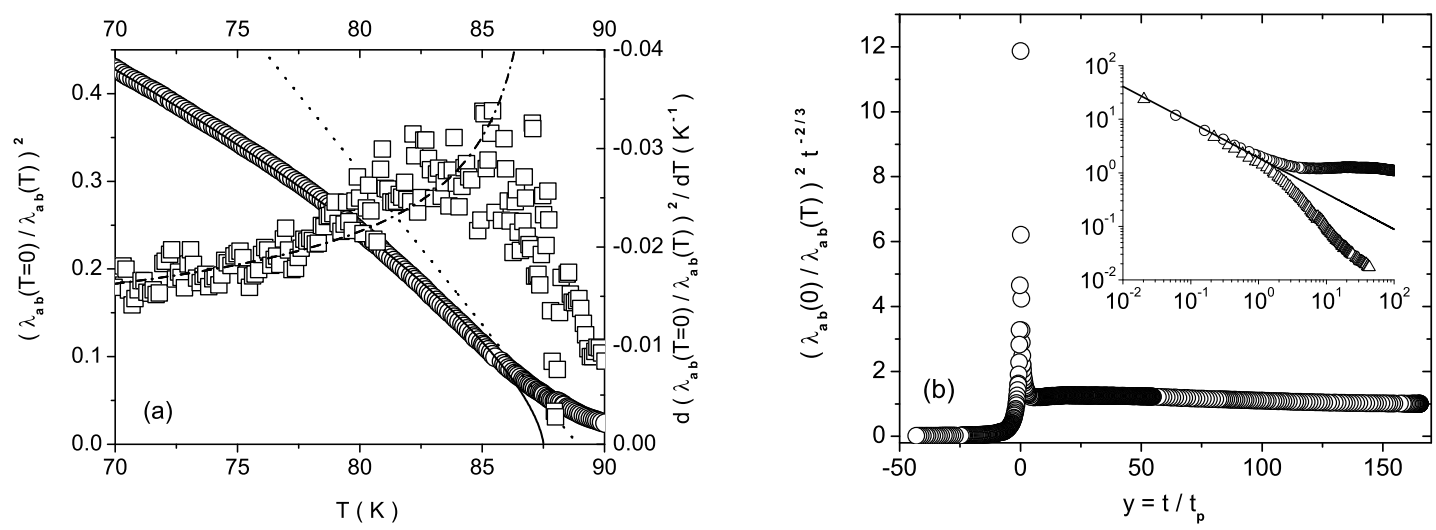

FIG. 5. (a)Microwave surface impedance data for $\lambda_{a b}^{2}(0) / \lambda_{a b}^{2}(T)(\bigcirc)$ and $d\left(\lambda_{a b}^{2}(0) / \lambda_{a b}^{2}(T)\right) / d T(\square)$ versus $T$ of a high-quality $\mathrm{Bi}_{2} \mathrm{Sr}_{2} \mathrm{CaCu}_{2} \mathrm{O}_{8+\delta}$ single crystal taken from Jacobs et al. [24]. The solid line is $\lambda_{a b}^{2}(0) / \lambda_{a b}^{2}(T)=1.2\left(1-T / T_{c}\right)^{2 / 3}$ and the dash-dot line its derivative with $T_{c}=87.5 \mathrm{~K}$, indicating the leading critical behavior of the homogeneous system. The dotted line is the tangent to the inflection point at $T_{p} \approx 87 \mathrm{~K}$, where $d\left(\lambda_{a b}^{2}(T=0) / \lambda_{a b}^{2}(T)\right) / d T$ is maximum; (b) Finite size scaling function $g(y)=\left(\lambda_{0 a b} / \lambda_{a b}(T)\right)^{2}|t|^{-\nu}$ versus $y=t /\left|t_{p}\right|$ for the data shown in Fig.5a. The solid line in the inset is Eq.(15) with $g_{0}=1.6$.

To substantiate the occurrence of a finite size effect we explore the consistency with the associated scaling function. In the present case it is defined in terms of

$$
\left(\frac{\lambda_{0 a b}}{\lambda_{a b}(T)}\right)^{2}|t|^{-\nu}=g(y), \quad y=\operatorname{sign}(t)|t|\left(\frac{L_{c}}{\xi_{0 c}^{-}}\right)^{1 / \nu}=\operatorname{sign}(t) \frac{|t|}{t_{p}} .
$$

For $t$ small and $L_{c} \rightarrow \infty$, so that $\pm y \rightarrow \infty$ it should tend to $g(y \rightarrow \infty)=1$ and $g(y \rightarrow-\infty)=0$, respectively, while for $t=0$ and $L \neq 0$ it diverges as

$$
g(y \rightarrow 0)=g_{0} y^{-\nu}=g_{0}\left(\frac{|t|}{t_{p}}\right)^{-\nu}
$$


so that in this limit $\left(\lambda_{0 a b} / \lambda_{a b}\left(T_{c}, L\right)\right)^{2}=g_{0}\left|t_{p}\right|^{\nu}=g_{0} \xi_{0 c}^{-} / L_{c}$. As expected, a sharp superconductor to normal state transition requires domains of infinite extent. Moreover at $t_{p}, y=1$ and $d\left(\lambda_{0 a b} / \lambda_{a b}(T, L)\right)^{2} / d t=0$. Accordingly, there is an inflection point at $t_{p}$. Since the scaling function $g(y)$ depends on the type of confining geometry and on the conditions imposed (or not, in the case of free boundaries) at the boundaries of the domains, this applies to the amplitude $g_{0}$ as well. In Fig.5b we displayed the finite size scaling resulting from the data shown in Fig.5a. In view of the fact that the data satisfies the limiting behavior of the finite size scaling function remarkably well, there is strong evidence for a finite size effect.

\section{FINITE SIZE AND ISOTOPE EFFECTS}

Recently we explored the effect of oxygen isotope exchange in $\mathrm{Y}_{1-x} \operatorname{Pr}_{x} \mathrm{Ba}_{2} \mathrm{Cu}_{3} \mathrm{O}_{7-\delta}$ on the inhomogeneity induced finite size effect by means of in-plane penetration depth measurements [10]. Defining the relative oxygen isotope shift of a physical quantity $X$ as $\left.\Delta X / X=\left({ }^{18} X-{ }^{16} X\right) /{ }^{16} X\right)$ we note that the shifts are not independent but according to Eq.(13) related by

$$
\frac{\Delta L_{c}}{L_{c}}=\frac{\Delta T_{p_{c}}}{T_{p_{c}}}+\frac{\Delta \lambda_{a b}^{2}\left(T_{p_{c}}\right)}{\lambda_{a b}^{2}\left(T_{p_{c}}\right)} .
$$

From the resulting estimates, listed in Table II The resulting estimates are summarized several observations emerge. First, $L_{c}$ increases systematically with reduced $T_{p_{c}}$. Second, $L_{c}$ grows with increasing $x$ and upon isotope exchange $\left({ }^{16} \mathrm{O},{ }^{18} \mathrm{O}\right)$. Third, the relative shift of $T_{p_{c}}$ is very small. This reflects the fact that the change of $L_{c}$ is essentially due to the superfluid, probed in terms of $\lambda_{a b}^{2}$. Accordingly, $\Delta L_{c} / L_{c} \approx \Delta \lambda_{a b}^{2} / \lambda_{a b}^{2}$ for $x=0,0.2$ and 0.3 . Indeed the relative shifts of $T_{p_{c}}, \lambda_{a b}^{2}\left(T_{p_{c}}\right)$ and $L_{c}$ are not independent.

\begin{tabular}{|c|c|c|c|}
\hline $\mathrm{x}$ & 0 & 0.2 & 0.3 \\
\hline$\Delta T_{p_{c}} / T_{p_{c}}$ & $-0.000(2)$ & $-0.015(3)$ & $-0.021(5)$ \\
\hline$\Delta L_{p_{c}} / L_{p_{c}}$ & $0.12(5)$ & $0.13(6)$ & $0.16(5)$ \\
\hline$\Delta \lambda_{a b}^{2}\left(T_{p_{c}}\right) / \lambda_{a b}^{2}\left(T_{p_{c}}\right)$ & $0.11(5)$ & $0.15(6)$ & $0.15(5)$ \\
\hline${ }^{16} \lambda_{a b}^{2}\left({ }^{16} T_{p_{c}}\right) /{ }^{16} \lambda_{a b}^{2}(0)$ & $4.4(2)$ & $4.0(2)$ & $4.4(2)$ \\
\hline${ }^{18} \lambda_{a b}^{2}\left({ }^{18} T_{p_{c}}\right) /{ }^{16} \lambda_{a b}^{2}(0)$ & $4.9(2)$ & $4.6(2)$ & $5.2(2)$ \\
\hline${ }^{16} T_{p_{c}}(K)$ & $89.0(1)$ & $67.0(1)$ & $52.1(2)$ \\
\hline${ }^{18} T_{p_{c}}(K)$ & $89.0(1)$ & $66.0(2)$ & $51.0(2)$ \\
\hline${ }^{16} L_{p_{c}}(\AA)$ & $9.7(4)$ & $14.2(7)$ & $19.5(8)$ \\
\hline${ }^{18} L_{p_{c}}(\AA)$ & $10.9(4)$ & $16.0(7)$ & $22.6(9)$ \\
\hline${ }^{16} \lambda_{a b}(0)(\AA)$ & $1250(10)$ & $1820(20)$ & $2310(30)$ \\
\hline \multicolumn{4}{|c}{}
\end{tabular}

Table II: Finite size estimates for ${ }^{16} T_{p_{c}},{ }^{18} T_{p_{c}},{ }^{16} \lambda_{a b}^{2}\left({ }^{16} T_{p_{c}}\right) /{ }^{16} \lambda_{a b}^{2}(0)$ and ${ }^{18} \lambda_{a b}^{2}\left({ }^{18} T_{p_{c}}\right) /{ }^{16} \lambda_{a b}^{2}(0)$, and the resulting relative shifts $\Delta T_{p_{c}} / T_{p_{c}}$ and $\Delta \lambda_{a b}^{2}\left(T_{p_{c}}\right) / \lambda_{a b}^{2}\left(T_{p_{c}}\right)$ for an ${ }^{18} \mathrm{O}$ content of $89 \% .{ }^{16} L_{p_{c}},{ }^{18} L_{p_{c}}$ and $\Delta L_{p_{c}} / L_{p_{c}}$ follow from Eq.(13). ${ }^{16} \lambda_{a b}(0)$ are $\mu$ SR estimates [29]

To appreciate the implications of these estimates, we note that for fixed Pr concentration the lattice parameters remain essentially unaffected [30,31]. Accordingly, an electronic mechanism, without coupling to local lattice distortions, implies $\Delta L_{c}=0$. On the contrary, a significant change of $L_{p_{c}}$ upon oxygen exchange uncovers the coupling to local lattice distortions involving the oxygen lattice degrees of freedom. A glance to Table I shows that the relative change of the domains along the c-axis upon oxygen isotope exchange is significant, ranging from 12 to $16 \%$, while the relative change of the inflection point or the transition temperature is an order of magnitude smaller. For this reason the significant relative change of $L_{c}$ at fixed $\operatorname{Pr}$ concentration is accompanied by essentially the same relative change of $\lambda_{a b}^{2}$, which probes the superfluid. This uncovers unambiguously the existence and relevance of the coupling between the superfluid, lattice distortions involving the oxygen lattice degrees of freedom. Potential candidates are the $\mathrm{Cu}-\mathrm{O}$ bond-stretching-type phonons showing temperature dependence, which parallels that of the superconductive order parameter [32]. Independent evidence for the shrinkage of limiting length scales upon isotope exchange stems from the behavior close to the quantum superconductor to insulator transition where $T_{c}$ vanishes [33]. Here the cuprates become essentially two dimensional and correspond to a stack of independent slabs of thickness $d_{s}[34,35]$. It was 
found that the relative shift $\Delta d_{s} / d_{s}$ upon isotope exchange adopts a rather unique value, namely $\Delta d_{s} / d_{s} \approx 0.03$ [33]. Although the majority opinion on the mechanism of superconductivity in the cuprates is that it occurs via a purely electronic mechanism involving spin excitations, and lattice degrees of freedom are supposed to be irrelevant, the relative isotope shift $\Delta L_{c} /{ }^{16} L_{c} \approx \Delta \lambda_{a b}^{2} /{ }^{16} \lambda_{a b}^{2} \approx 0.15$ uncovers clearly the existence and relevance of the coupling between the superfluid, lattice distortions and anharmonic phonons which involve the oxygen lattice degrees of freedom.

In contrast to $\mathrm{YBa}_{2} \mathrm{Cu}_{3} \mathrm{O}_{7-\delta}$, $(\mathrm{K}, \mathrm{Ba}) \mathrm{BiO}_{3}, \mathrm{MgB}_{2}, 2 \mathrm{H}-\mathrm{NbSe}_{2}$ and $\mathrm{Nb}_{77} \mathrm{Zr}_{23}$, where the lower bounds for the length scale $L$ of the superconducting grains ranges from $182 \mathrm{~A}$ to $814 \AA$, we have seen that the data for $\mathrm{Bi}_{2} \mathrm{Sr}_{2} \mathrm{CaCu}_{2} \mathrm{O}_{8+\delta}$ single crystals and $\mathrm{Y}_{1-x} \mathrm{Pr}_{x} \mathrm{Ba}_{2} \mathrm{Cu}_{3} \mathrm{O}_{7-\delta}$ uncovers the existence of nanoscale inhomogeneities, which are not merely an artefact of the surface, but a bulk property with spatial extent, giving rise to finite size effects and with that to a rounded thermodynamic phase transition which occurs smoothly. While there are many questions to be answered about the intrinsic or extrinsic origin of the inhomogeneities, the existence and the nature of a macroscopic superconducting state, we established that a finite size scaling analysis yields the spatial extent of the inhomogeneities. Furthermore, we established that the change of the spatial extent of the inhomogeneities upon oxygen isotope exchange uncovers the relevance of lattice degrees of freedom in mediating superconductivity.

\section{ACKNOWLEDGMENTS}

The author is grateful to R. Khasanov, H. Keller and K.A. Müller for very useful comments and suggestions on the subject matter.

[1] G. Bednorz and K. A. Muller, Z. Phys. B 64, 189 (1986).

[2] J. Mesot, P. Allensbach, U. Staub and A. Furrer, Phys. Rev. Lett. 70, 865 (1993).

[3] A. Furrer et al., Physica C 235-240, 261 (1994).

[4] J. Liu, J. Wan, A. Goldman, Y. Chang and P. Jiang, Phys. Rev.Lett. 67, 2195 (1991).

[5] A. Chang, Z. Rong, Y. Ivanchenko, F. Lu and E. Wolf, Phys. Rev. B 46, 5692 (1992).

[6] T. Cren, D. Roditchev, W. Sacks, J. Klein, J.-B. Moussy, C. Deville-Cavellin, and M. Laguës, Phys. Rev. Lett. 84, 147 (2000).

[7] K. M. Lang, V. Madhavan, J. E. Hoffman, E. W. Hudson, H. Eisaki, S. Uchida and J. C. Davis, Nature 415, 413 (2002).

[8] Ch. Renner and Ø. Fischer, Phys. Rev. B 51, 9208 (1995).

[9] T. Schneider, cond-mat/0210702.

[10] T. Schneider, R. Khasanov, K. Conder and H. Keller, cond-mat/0302043.

[11] M. E. Fisher, in Critical Phenomena, Proceedings of the 1970 International School of Physics Enrico Fermi,Course 51, edited by M. S. Green (Academic, New York, 1971), p. 1.

[12] A. Peliasetto and E. Vicari, cond-mat/0012164.

[13] M. Roulin, A. Junod and E. Walker, Physica C 296, 137 (1998).

[14] A. Mirmelstein, A. Junod, E. Walker, B. Revaz, Y.-Y. Genoud, and G. Triscone, J. Superconductivity 10, 527 (1997).

[15] D. Sanchez, A. Junod, J. Muller, H. Berger and F. Levy, Physica B 204,167 (1995).

[16] L. Lyard et al., cond-mat/020623.

[17] S. Blanchard et al., Physica C 369, 193 (2002).

[18] A. Junod, A. Erb and Ch. Renner, Physica C 317-318, 333 (1999).

[19] A. Carrington et al., Phys. Rev. B55, 8674 (1997).

[20] B. Janossy, D. Prost, S. Pekker and L. Fruchter, Physica C 181, 91 (1991).

[21] T. Schneider and J. M. Singer, Physica C 341-348, 87 (2000).

[22] A. Mirmelstein, A.Junod, G.Triscone, K.-Q.Wang and J.Muller, Physica C 248, 335 (1995).

[23] A. Junod et al., Physica C 229, 209 (1994).

[24] T. Jacobs, S. Sridhar, Q. Li, G. D. Gu, N. Koshizuka, Phys. Rev. Lett. 75, 4516 (1995).

[25] T. Schneider and J. M. Singer, Phase Transition Approach To High Temperature Superconductivity, Imperial College Press, London, 2000, p. 186.

[26] P. C. Hohenberg, A. Aharony, B. I. Halperin and E. P. Siggia, Phys. Rev. B 13, 2986 (1976).

[27] K. D. Osborn, D. J. Van Harlingen, Vivek Aji, N. Goldenfeld, S. Oh and J. N. Eckstein, cond-mat/0204417.

[28] S. F. Lee et al., Phys. Rev. Lett. 71, 3862 (1993).

[29] R. Khasanov et al., J. Phys. Condensed Matter 15, L17 (2003). 
[30] K. Conder et al., in Phase Separation in Cuprate Superconductors, edited by E. Sigmund and K. A. Muller (Springer, Berlin 1994) p. 210.

[31] F. Raffa et al., Phys. Rev. Lett. 81, 5912 (1998).

[32] J.-H. Chung et al., Phys. Rev. B 67, 014517 (2003).

[33] T. Schneider, Phys. Rev. B 67, 134514 (2003).

[34] T. Schneider, Europhys. Lett. 60, 141 (2002).

[35] T. Schneider, Physica B 326, 289 (2003). 